\title{
Suppressed HF Behaviour in the Periodic Anderson Model
}

\author{
Okunzuwa I. S. \\ Arthur I. I. Ejere \\ Department of Physics, Faculty of Physical Sciences, \\ University of Benin, Benin city, Nigeria
}

Doi: 10.19044/esj.2018.v14n12p241 URL:http://dx.doi.org/10.19044/esj.2018.v14n12p241

\begin{abstract}
The periodic Anderson model is applied to 4 electrons on 4 sites with periodic boundary conditions. We applied magnetic field to the localized forbitals, $E_{\sigma f}$. The number of electrons is taken to be one per site and the interactions between different sites is restricted to nearest neighbors. The many body eigenvalues are calculated exactly using exact diagonalization technique. We find that the specific heat is suppressed by the variation of the band energy of the localized f-orbitals as mediated by the application of the magnetic field, $\mathrm{H}$, under various hybridization energy. A continuous suppression of the specific heat reduces the heavy fermion behavior in the system.
\end{abstract}

Keywords: Average energy, Ferromagnetism, Ground State energy, partition function, specific heat

\section{Introduction}

Many rare-earth and actinide compounds have been known over the decade to exhibit mixed valence phenomena [Batista et al, 2003]. The valence fluctuation in such systems are highly correlated, they are also found to exhibit Fermi-liquid phenomena with a heavy mass at low temperature. There is also increasing interest in these heavy fermion materials. The common feature of these heavy fermion systems is that at low temperatures they exhibit large values of specific heat coefficient [Batista et al, 2003].

There has been extensive theoretical work on mixed valence (MV) and Kondo systems [Batista et al, 2003, Chabe et al, 2008]. However, the Kondo problem and the MV regime are well understood. The Anderson Model has been used to extensively study the thermodynamic, transport and excitation properties of these systems using some powerful techniques [Herin et al, 2007]. 
However, the lattice problem in these regimes is neither well understood nor solved. There is need to also explain the wide range of physical properties.

A Fermi liquid theory which discards quasi particle interactions has been developed [Jarrell, 1995]. It is found that the large effective mass of HF systems does not arise primarily due to band renormalization and hence contradict many theories [Chabe et al, 2008]. From the foregoing remarks, it is obvious that there remain a need for greater understanding of the MV and by extension the HF systems.

Though, sometimes small clusters may not represent bulk materials. However, the large number of states obtained even in small clusters statistically may give results which fairly represent a large system over a reasonable temperature range [Sordi et al, 2007]. In the present work, the periodic Anderson Model is applied to a chain of four electrons on four sites with period boundary conditions. There would be a systematic study of the effect of varying energy of the localized $f$-orbital mediated by the application of magnetic field to the localized orbital only. One extended orbital would be considered per site per spin with an inter-atomic transfer integral $t$. There is also one localized $f$-orbital per site per spin having energy $\mathrm{E}_{\mathrm{f}}$ with coulombic repulsion $u$ between two electrons in the $f$ orbitals on the same site. An hybridization term $\mathrm{v}$, between the localized and extended orbitals is also present in the model. One electron per site would be allowed, and it is restricted to nearest neighbours. An exact diagonalization technique would be applied to generate the matrix. The matrix would be diagonalized using the mathematical 9 wolfram program.

The ground state is a singlet $(\mathrm{S}=0)$ while the other states are excited $(\mathrm{S}>0)$ and are nearly degenerated with the ground state. The specific heat increases sharply at low temperatures which is comparable to the heavy fermion behaviour. The systematic variation of the energy of the f-orbital and its corresponding effect on the specific heat of the heavy fermion system would be carried out.

\section{Methodology}

We use the Anderson Lattice model (AL) [Yu et al, 2008a] which is a translationally invariant generalization of the Anderson impurity model which has been successfully used to explain the Kondo effect.

The Anderson Lattice (AL) Hamiltonian in second quantization is given by:

$H=\sum_{i j \sigma} t_{i j} c_{i \sigma}^{+} c_{j \sigma}+\sum_{i \sigma} E_{\sigma f} f_{i \sigma}^{+} f_{i \sigma}+{ }^{v} / \sqrt{N} \sum_{i \sigma}\left(f_{i \sigma}^{+} c_{i \sigma}+H . C\right)+u \sum_{i \cdot} n_{i \uparrow}^{f} n_{i \downarrow}^{f}$

Where $t$ is the energy of the conduction band. $\mathrm{v}$ is the hybridization 
interaction between the conducting band electrons and the localized felectrons. The onsite coulomb repulsion is $U$ and $E_{\sigma f}$ is the eigenvalue for a single f-electron. The local spin does not flip in the Anderson Model. Because of the hybridization term, a local spin that is down can become a conduction state with spin down, and then wander away [Sordi et al, 2007]. Later, a different conduction electron with spin up can come and reside in the local orbital.[ Hywel ,2008] This process gives the appearance of the local spin having flipped from up to down.

Furthermore, $E_{\sigma f}=E_{f}-\sigma h, h=\mu_{B} H$ and $H$ denotes the magnetic field.

The ground state energy is obtained using the exact diagonalization method and the model (i.e. eqn. 1) is studied under cyclic boundary conditions. This case has the advantage of eliminating edge effect. We use the Wolfram Mathematica 9 Software [Wolfram Research, Inc (2007] which is effective in diagonalizing large matrix sizes. With these tools, we were able to study systems of 4 electrons on 4 sites for which the Hamiltonian matrix size is 70 $\mathrm{x} 70$. Computing the ground state energy, using

$$
E=\frac{\langle\psi|H| \psi\rangle}{\langle\psi \mid \psi\rangle}
$$

Where we have $E=\frac{1}{2}\left[u+\sqrt{16 t^{2}+u^{2}}+2 \theta\right]$

and $\theta=2 E_{\sigma f}+2 v / \sqrt{N}$

Calculating the properties of the system of particular interest is the partition function $\mathrm{Z}$ of the electrons. When a grand-canonical ensemble is considered we can deduce a number of static thermodynamic properties. The partition function is the trace of an operator Z [Zhu. and Zhu 2001].

$Z=\operatorname{Tr}\langle i|\exp (-\beta H)| j\rangle$

Where $\beta=1 / k T$

and $H$ is Hamiltonian matrix.

Hence, the partition function of the system under consideration is given by equation (6)

$Z=2 \exp (-6 \alpha)+4 \exp (-6 \theta)$

Where $\alpha=\theta+u$

We define the expectation value $\langle H\rangle=E$ as the operator for $H$. That is

$E=\langle H\rangle=\frac{1}{Z}\langle i|H \exp (-\beta H)| j\rangle$

Equation (7) describes the thermal average of the quantity $\mathrm{H}$. This can be used to characterize the electron correlation [Galpin et al, 2008]].

Therefore $E=\langle H\rangle=\frac{(\theta+u) \exp (-\beta(\theta+u))+2 \theta \exp (-\beta \theta)}{\exp (-\beta(\theta+u))+2 \exp (-\beta \theta)}$

The temperature dependence of the thermodynamic, quantity (specific 
heat) is calculated from the many body eigenvalue (the ground state energy) by using the standard statistical mechanics procedure for a canonical ensemble. The expression for specific heat is given by equation 10. Our result is expressed in units of $\mathrm{J} / \mathrm{mol}-\mathrm{k}$, where it is understood that 'mole' means $\mathrm{N}$ electrons.

$C_{v}=\frac{\partial E}{\partial T}$

$C_{v}=\frac{\gamma-\Sigma}{\Omega}$

Where

$r=[\alpha \exp (-\alpha b)+2 \theta \exp (-\Theta b)]\left[\frac{\alpha}{K} \exp (-\alpha b)+\frac{2 \theta}{K} \exp (-\theta b)\right]$

$\Sigma=[\exp (-\propto b)+2 \exp (-\Theta \wp)]\left[\left(\alpha^{2} / K\right) \exp (-\propto b)+\left(2 \theta^{2} / K\right) \exp (-\Theta b)\right]$

$\Omega=\exp (-2 \alpha b)+4 \exp (-2 \theta b)+4 \exp (-\alpha+\theta) b$

\section{Result and discussion}

We discuss the temperature dependence of the specific heat for a solution of values of $E_{\sigma f}$ and $v / t /$ chosen from a large set of available results to represent interesting cases. We found that the specific heat curves are qualitatively similarly for different values of $E_{\sigma f .}$. Figures 1 and 2 are plotted on a common linear scale of temperature $T / t /$, hybridization $\mathrm{v}$, respectively. If $t$ is set to $1 \mathrm{ev}$, then the scale is in Kelvin.

Firstly, we consider the symmetric case, $\mathrm{E}_{\mathrm{f}}=-\mathrm{u} / 2$. We fix $t=1, u=$ 1.0, $H=0.01$ and $v$ vary from 0.1 to 1.0 (all energies are in units of $t$ ). When the hybridization is relatively large $(\mathrm{v}=0.1$ to 1.0$)$ no peak structure is obtained in the temperature range of interest to us. This indicate the absence of the Schottky type behavior as there is no peak observed for $E_{\sigma f}=-5.0$ and $v$ increases from 0.1 to 1.0. This indicates the absence of degeneracy [8].

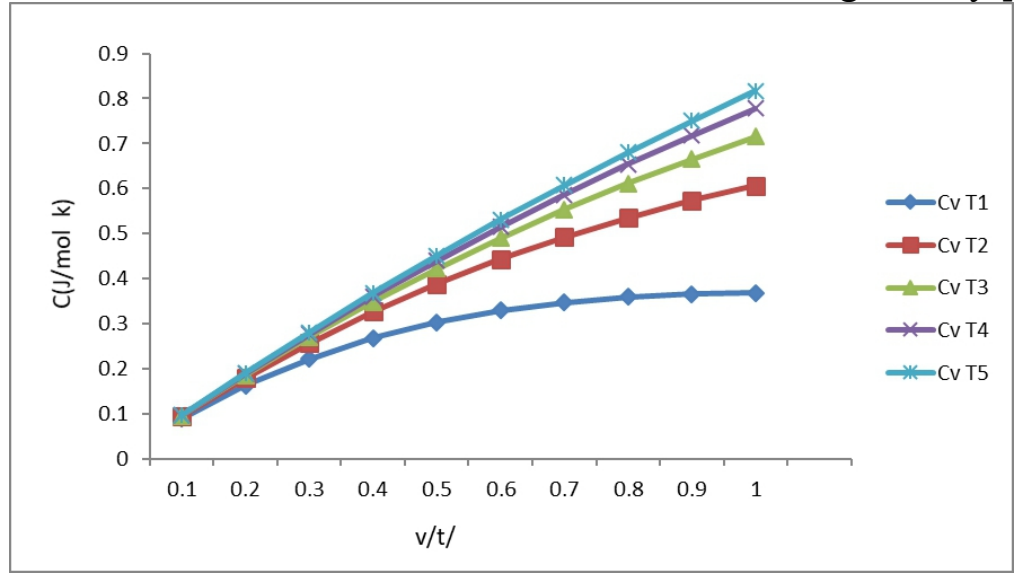

Fig 1: $\mathrm{C}_{\mathrm{v}}$ vs $\mathrm{v}$ for various $\varepsilon_{\text {of }}$ for $\mathrm{t}=1, \mathrm{H}=0.01, \mathrm{u}=1$ 
From the ranges of values obtained from $E_{\text {of }}$ ranging from -0.5 to -0.4 shows that the Specific Heat ( $\mathrm{SH})$ resembles that of a Kondo system. Under very low temperature $(\mathrm{T}=1 \mathrm{k})$ the $\mathrm{SH}$ shows a near linear increase as the hybridization increases which is indicated by $C_{v}(T 1)$ in Fig. 1. It is also observed that this trend changes slightly as temperature is gradually increased until a broad peak is reached at $T=5 k$ as indicated by $C_{v}(T 5)$. This behavior can be explained in the following way: when the hybridization $v$, gradually increases for $E_{\text {of }}=-0.5$, the ' $\mathrm{f}$ ' level are well defined and essentially full $\left(\mathrm{n}_{\mathrm{f}} \sim\right.$ 1) at low temperatures. Excitations occur giving rise to a low temperature linear behavior of $\mathrm{SH}$.

Secondly, our results indicate that the SH increases very rapidly at low temperatures. The unusual rise mimics the onset of the HF behavior. In order to study this in more details, we present a typical example of a tetrahedron for $t=1, u=1, H=1.0, v=0.1$ and plot in Fig. $2, \mathrm{Cv} / \mathrm{T}$ against $\mathrm{T}$ for $E_{\text {of }}$ ranging from -0.5 to -0.4 .

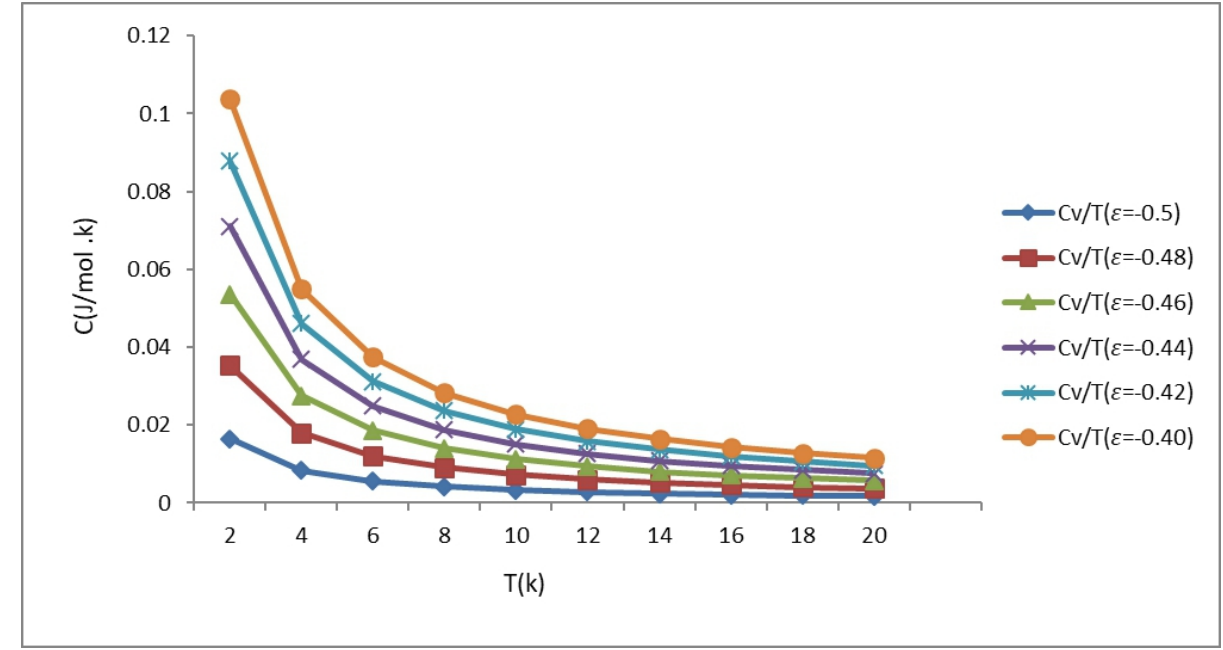

Fig. 2: $\mathrm{C}_{\mathrm{v}} / \mathrm{T}$ vs $\mathrm{T}$ for various $\varepsilon_{\sigma \mathrm{f}}$ for $\mathrm{t}=1, \mathrm{H}=1, \mathrm{v}=0.1, \mathrm{u}=1$

We notice that the rate of increase of $\mathrm{C}_{\mathrm{V}} / \mathrm{T}$ (at very low temperatures) is maximum for $E_{\sigma f}=-0.4$. Again, for each $E_{\sigma f}$, the ground state increases. The ground state is a singlet. The next higher energy states are well separated from this ground state. The low-temperature rise in SH is determined by the separation of these levels. As $E_{\sigma f}$ increases, the separation between the ground state and other excited states increases, and the rise in $\mathrm{C} / \mathrm{T}$ correspondingly increases. Thus, we notice that the HF behaviour is obtained when the many body ground state is a singlet. The electrical resistance of a pure metal drops as temperature is lowered. However, the resistance saturates as the temperature is lowered below about 10k. This behaviour changes dramatically when magnetic atoms such as Cobalt, are added. Rather than saturating, the electrical resistance increases as the temperature is lowered further. The so- 
called Kondo temperature-roughly speaking the temperature at which the resistance starts to increase again -completely determines the low temperature electronic properties of the material [Kubo, 2015]. This HF behaviour, however is affected by the application of magnetic field $(\mathrm{H})$. The resistivity of the PAM with magnetic field applied to the localized orbitals is obtained from eqn. 14 below.

$\ln Z=-1 / k T\left[2\left(\varepsilon_{F}-\frac{\mu H}{\rho}\right)+\frac{2 v}{\sqrt{N}}+u\right]-2 / \mathrm{kT}\left[2\left(\varepsilon_{F}-\frac{\mu H}{\rho}\right)+\frac{2 v}{\sqrt{N}}\right]$

Hence,

$$
\rho=6 \mu H \sqrt{N} /\left(6 \varepsilon_{f} \sqrt{N}+6 v+u \sqrt{N}+k T \ln Z \sqrt{N}\right.
$$

The graph of $\rho$ plotted for various $\varepsilon_{\sigma f}$ for $\mathrm{H}=1.0, \mathrm{u}=1.0, \mathrm{v}=1.0$ is given in fig. 3 below.

From fig. 3 and with $\mathrm{H}$ only acting on the localized orbital results in a downturn of the resistivity, $\rho$ at temperatures below $5 \mathrm{k}$. This downturn indicates a suppressed HF behaviour.

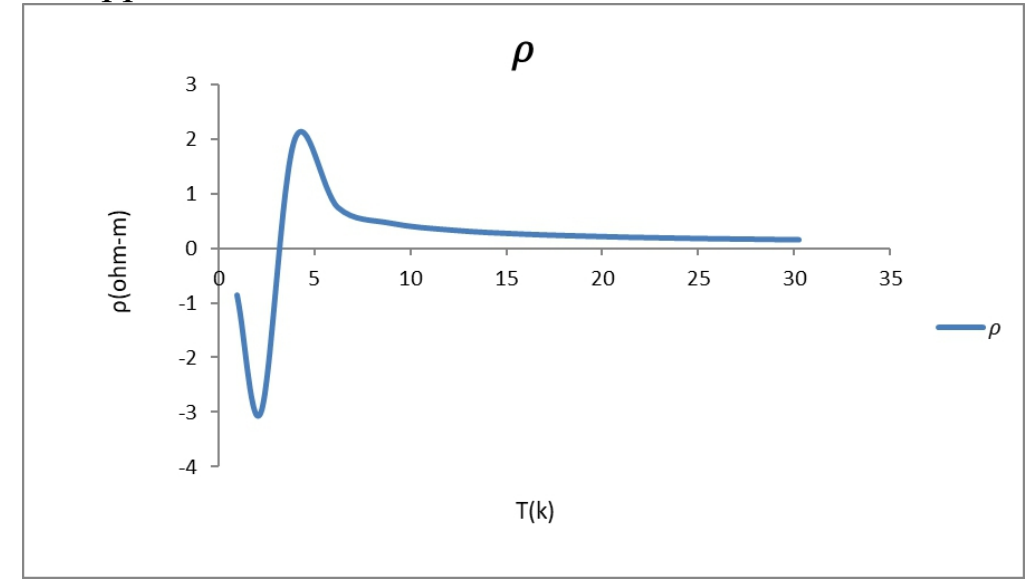

Fig. 3: The graph of $\rho$ plotted for various $\varepsilon_{\sigma f}$ for $\mathrm{H}=1.0, \mathrm{u}=1.0, \mathrm{v}=1.0$

\section{Summary and conclusion}

We have calculated the thermodynamic property (specific heat) for a range of parameters $U, V$ and $E_{\text {of }}$ and the resistivity for various values of localized band energies by using the Periodic Anderson Model. Our most important conclusion is that calculation are capable of reproducing the types of SH behavior observed in Heavy Fermions and Kondo systems. An important parameter in determining the SH property it was found that as $\mathrm{E}_{\text {of }}$ is increased, there was a broad peak observed at low temperature $\mathrm{T}=1 \mathrm{k}$. A striking feature of our results is the occurrence of HF behavior. We find that when $E_{\sigma f}$ is large and negative for very low values of $v$, the many body ground state is a singlet. The other excited states have much higher energy. The specific heat rises sharply at low temperatures and the system exhibits HF features. 
We conclude that at low temperatures, the SH deeps as $v$ reduces. With the application of magnetic field of $H=1.0$, the energy of the localized band $E_{\sigma f}$ is varied. This affects the $\mathrm{SH}$ and the resistivity as they are suppressed by the activities of the ' $\mathrm{f}$ ' electrons. It is therefore concluded, that as $E_{\sigma f}$ increases further, the system would leave the HF regime.

\section{References:}

1. Batista C.D., Bonca J., and Gurbernatis J.E. (2003). Ferromagnetism in the Two-Dimensional Periodic Anderson Model. Phys. Rev. B 63: 184426 - 184439; Retrieved from http/www.arXiv: condmat/0009128.

2. Chabe J., Lamarie G., Gremaud B., Delande D., Szriftgiser P. and Garreau J.C. (2008). Experimental Observation of the Anderson Transition with Atomic Matters Waves. Phys. Rev. Lett. 101. No. 25, $255702-255706$.

3. Herin M., Scheffler M., Dressel M., and Hilbert L. (2007). Signature of Electronic Correlations in the Optical Conductivity of the Doped Semiconductor Si: P. Phys. Rev. B 75. 205203 - 205212.

4. Jarrell M. (1995). Symmetric Periodic Anderson on infinite Dimensions. Phys. Rev. B. 51, $7429-7440$.

5. Sordi G. Amaricci A., and Rozenberg M.J. (2007). Metal-Insulator Transitions in the Periodic Anderson Model. Phys. Rev. Lett., 99, 196403 - 196407.

6. Yu Unjong, Byczuk K., and Volldhardt D. (2008a). Influence of band and Orbital Degeneracies on Ferromagnetism in the Periodic Anderson Model. Phys. Rev. B. 78: 205118 - 205122; Retrieved from http/www.arXiv:condmat/0808.0079.

7. Wolfram Research, Inc (2007). Mathematics 6.0 Software. Champaign, Illinois, U.S.A.

8. Zhu L. and Zhu J. (2001). Magnetic Field Induced Phase Transitions in the Two-Impurity Anderson Model. Submitted to Phys. Rev. Lett. Retrieved from http/www.arXiv1011.6629v1 [cond-mat.str-el].

9. Robert G. Hywel (2008). Magnetism and Transport in Nanostructure Domain Wall Systems. PhD Thesis Submitted to the Department of Physics, University of Bath, U.K.

10. Galpin R. Martin and Logan E. David (2008). Anderson Impurity Model in a Semiconductor. Phys. Rev. B. 77: 195108 - 195114; Retrieved from http/www.arXiv:cond-mat/0902.433

11. katsunori Kubo (2015). Variational Monte Carlo study of magnetic states in periodic Anderson model. Journal of Physics: conference Series 592012039 